\title{
Using Sandtray Integrating Discrimination Model in Counseling Supervision: An Initial Empirical Analysis
}

Mei-Hsiang Tsai, Department of Counseling and Guidance, National University of Tainan, Taiwan

Mon-Hsin W Flahive, Department of Counseling Psychology, Chinese Culture University, Taiwan

\section{INTRODUCTION}

The purpose of the present research aims to explore the impact of sandtray integrating discrimination model on supervision efficacy and supervisee's anxiety.

Before conducting clinical sandtray supervision, the supervisors received training on sandtay therapy for two days, discrimination supervision model for two days, and sandtray integrating discrimination supervision model for two days, with a total of six days. Once training is completed, the supervisor conducted weekly individual sandtray supervision with his or her paired supervisee, with a total of 12 counseling supervision sessions.

In addition, the supervisors complete the $\ulcorner$ Sandtray Supervision Self-

Evaluation Form $\lrcorner$ after conducting sandtray supervision, while the supervisees fill out the $\ulcorner$ Anticipatory Supervisee Anxiety Scale 」 before receiving sandtray supervision.

Based on the results, it is expecting to acknowledge the impact and efficacy of the sandtray supervision model and its application. It is also expecting to establish the fundamental research for future expressive individual supervision model.

\section{METHOD}

\section{Participants}

The current study recruited five paired counseling supervisors and full-time counseling interns from three university counseling centers in Taiwan.

Table 1 Participants' Background Information

\begin{tabular}{|l|c|c|c|}
\hline Participants & \multicolumn{1}{c}{ ID } & SEX & AGE \\
\hline Supervisor & SRA & F & $30-35$ \\
\hline & SRB & M & $35-40$ \\
\hline & SRC & F & $35-40$ \\
\hline & SRD & F & $35-40$ \\
\hline & SRE & F & $40-45$ \\
\hline Supervisee & SEA & M & $26-30$ \\
\hline & SEB & F & $26-30$ \\
\hline & SEC & F & $26-30$ \\
\hline & SED & F & $26-30$ \\
\hline & SEE & F & $26-30$ \\
\hline
\end{tabular}

Instruments

Two instruments are used in this study:

1. Sandtray Supervision of Self-Evaluation Form

2. Anticipatory Supervision Anxiety Scale $>$ Data Analysis

All instrument data is analyzed using SPSS software to determine supervision efficacy and supervisee's anxiety level.

\section{RESULTS}

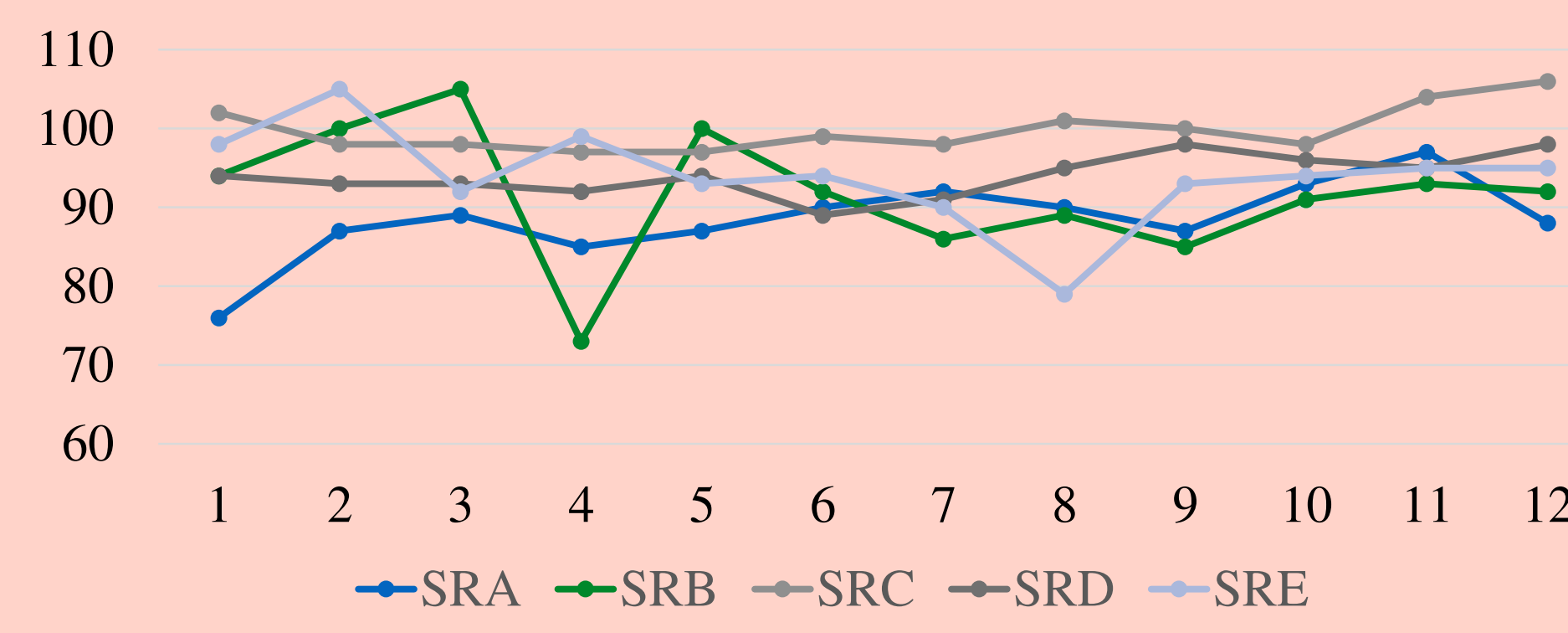

Figure 1 Sandtray Supervision of Self-Evaluation Form

120

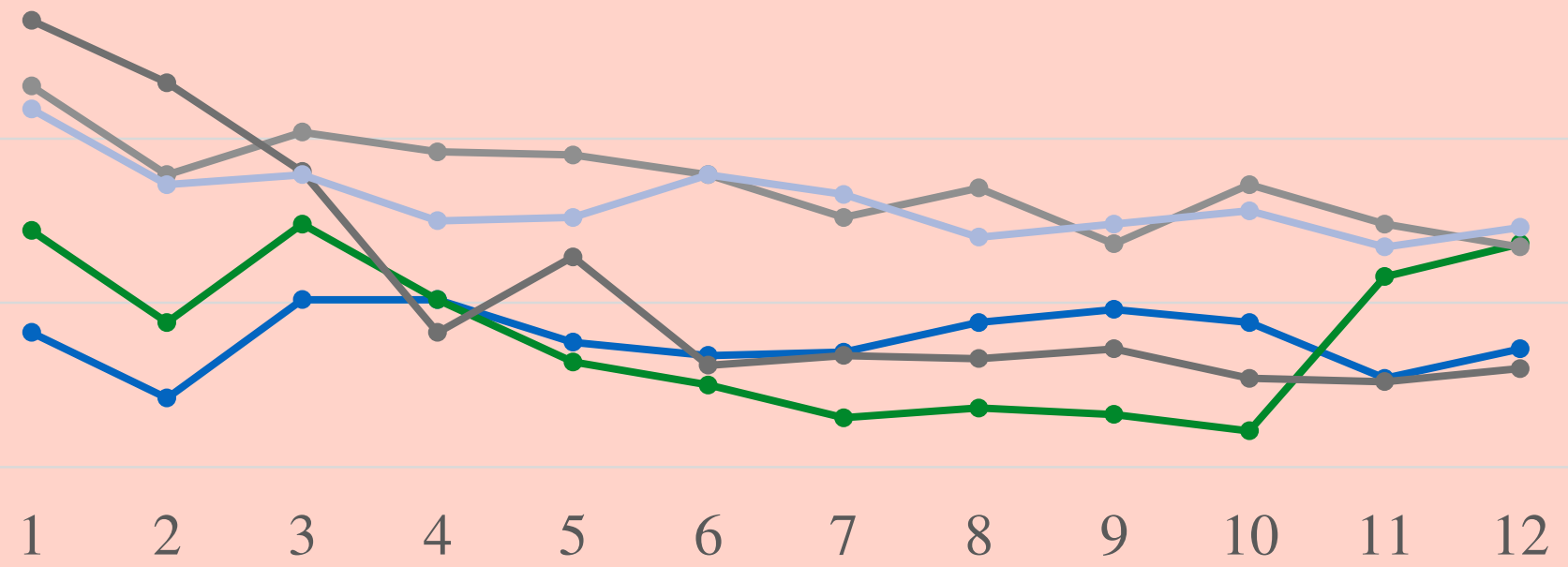

$\rightarrow$ SEA $\rightarrow$ SEB $\rightarrow$ SEC $\rightarrow$ SED $\rightarrow$ SEE

Figure 2 Anticipatory Supervision Anxiety Scale

Based on the results, the mean scores of supervisors' sandtray supervision efficacy self-evaluation on session 1 and session 12 are 92.8 $(\mathrm{SD}=9.96)$ and $95.8(\mathrm{SD}=6.79)$, respectively.

According to the findings, the mean scores of supervisees' anticipation anxiety level on sandtray supervision session 1 and session 12 are 114.8 $(\mathrm{SD}=37.957)$ and $74.8(\mathrm{SD}=20.142)$, respectively. 